\title{
Neural Processing Unit based on the Optical Bistable Properties of Semiconductor Laser Amplifiers
}

\author{
A.P. González-Marcos, J.A. Martín-Pereda \\ E.T.S. Ingenieros de Telecomunicación. \\ Universidad Politécnica de Madrid \\ 28040 Madrid, Spain
}

\begin{abstract}
This paper analyzes the behavior of a neural processing unit based on the Optical Bistable Properties of Semiconductor Laser Amplifiers. A similar unit to the reported here was previously employed in the simulation of the Mammalian Retina. The main advantages of the present cell are its larger fan-out and the possibility of different responses according to the light wavelength impinging onto the cell. These properties allow to work with larger structures as well as to obtain different behaviors according to the light characteristics. This new approach gives a possible modeling closer to the real biological configurations. Moreover, a more detailed analysis of the basic cell internal behavior is reported.
\end{abstract}

Keywords - Neural processing, optical bistability, neuronal modeling.

\section{INTRODUCTION}

Most of the intensity sensations in living bodies are related with absolute or relative thresholds for sensations. Neural responses follow, in almost every case, a no linear relation with the intensity of the external input. Moreover, in certain cases, the responses are of periodic type, for instance in the case of action potentials, being the frequencies of these responses strongly dependent on the intensity of the input stimulus. These facts have been the basis for most of the neural networks developed until now and constitute a very well established body of research and development. Since the pioneering work by Hopfield [1], where a large number of possible strands were indicated, the existing literature in this field is huge and the number of published papers increases every month. Both, theoretical and practical studies have been reported and the applications in many different fields are a proof of its validity.

Practical implementations of neural networks have been based, in most of the cases, on microelectronic structures where the non linear properties of semiconductor devices have been the origin of the behaviour of these networks. This solution has the advantage of working with devices whose properties are very well known and easily available. Moreover, it is possible to get a high level of integration. As a consequence, if we consider the number of incorporated units, the similarity between the implemented artificial structure and the biological one, may be very high. Finally, because the parameter values of the employed semiconductor devices cover a large range, a large variety of simulations may be covered. But there is a situation in which semiconductor devices are not the most suitable devices to be employed. This situation is related with the possibility to work in a parallel way. When parallel structures need to be employed the only possibility is to arrange a large number of parallel physical routes, normally integrated semiconductor doped paths. This solution has some disadvantages most of them related with the parasitic capacitances appearing between paths. Moreover, the number of possible parallel paths in any physical structure is limited by the possible level of integration as well as by other physical parameters of the component, namely, speeds and delay times. The solution to this problem has been the introduction of optical structures where a parallel working possibility is present. This solution has been offered by several authors [2], and a large number of proposals since then have been reported. The main problem with this solution is the small possible fan-out offered by most of the reported implementations. Because the output of any one of the employed optical devices has to go, in every case, to several new devices, the available optical power decreases as the number of devices increases. This is an important limitation to their use in large and complex parallel architectures.

A possible improvement of these works will be reported in this paper. It is based on the use of the optical bistable characteristics of semiconductor laser amplifiers and their application as building elements of Optically Processing Logic Cells, OPLCs, as the ones previously employed by the authors in the mammalian retina simulation. These devices offer the possibility to work with strong non linearities as well as to have optical outputs with optical powers as high as needed, as a function of the number of following steps. We will present, in the next section, a panoramic view of the properties of these structures and, in the last one, we will offer some details of their behaviour when employed in the simulation of biological sensing structures. 


\section{SEMICONDUCTOR LASER AMPLIFIERS AS BASIC} UNITS FOR MODELING SENSING STRUCTURES

Laser diode bistability has been studied from the very beginning of the Optical Bistability analysis, in the decade of the eighties. Bistable lasers have been proposed for fast switching as early as in 1986 [3]. The simplest configuration to be employed appears in Fig. 1. The first one (a) corresponds to a classical structure, acting the laser diode as laser amplifier. The signal to be employed impinges onto the active region of a laser diode through its frontal surface. Two possible outputs may appear. The first one (a) employs the transmitted light, in a similar way to the output from an optical amplifier. A small part will go out from the input surface. The use of this light corresponds to the second possible configuration. This working structure (Fig. 1.b) is called "reflective mode". In both cases, the characteristics of the output light will be dependent on the relation between the parameters of input light, namely power and optical frequency, and the characteristics of the laser diode acting as sensor, bias current and proper optical frequency.

The second possibility appears in Fig. 2. In this case, the device has two active sections separated by a gap. To turn on the first section as a laser, a threshold current is required. Once the laser is turned on, the output light pass through the gap and goes into the second active section. By providing a proper injection current to this second section, making its gain higher than the loss from the middle gap, the light feedbacks to the first section and provides a positive gain to this first section. As a result, the laser can still be on though the injection current is reduced below the threshold. The laser has a turn-off current smaller than the turn-on current. When the first section is biased at a current between these two values, the device is bistable. To change the state from one to another, either a reset current pulse or a set light pulse can be used. This type of structure is, basically, similar to the cleaved coupled-cavity semiconductor lasers, $\mathrm{C}^{3}$ lasers. Moreover, because the frequency of this type of lasers can

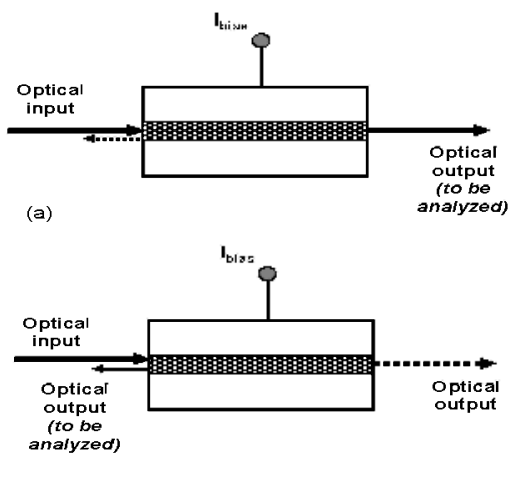

(b)

Figure 1.- Basic configurations for single laser diode sensors, (a) by transmission, and (b) by reflection.

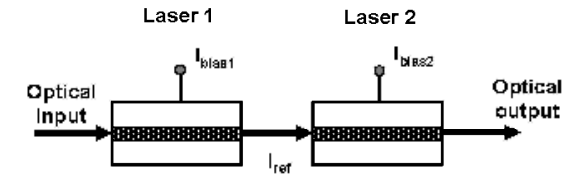

(a)

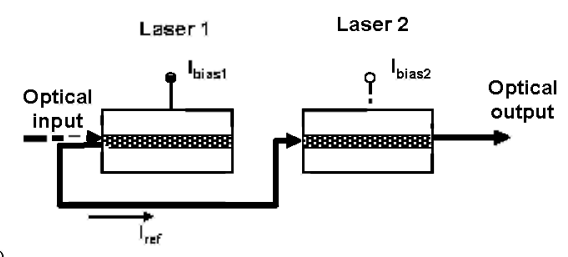

Fig. 2.- Possible configurations for feedback in double laser structures, (a) with transmitting signal, and (b) with reflecting signal from the first laser.

be tuned, it is possible design systems with them involving multichannel optical frequency as well as they allow new approaches to optical data switching and routing. In these lasers, the output spectrum becomes extremely pure as the only longitudinal mode that occurs near the peak of the gain can survive. As the injection current in the second diode varies by a small amount, the wavelength of the whole laser is shifted by exactly one Fabry Perot mode spacing, which is about $1,5 \mathrm{~nm}$, depending on the cavity length. This type of stepwise tuning continues over a very large spectral width of the gain profile, around $15 \mathrm{~nm}$. The corresponding configuration appears in Fig. 2.a

The above indicated working method is not the only possible one. The indicated configuration corresponds to a situation where the light entering the second laser is the obtained from transmission in the first laser. But feedback is possible too if the reflected light from the frontal surface of the first laser goes to the second laser. This situation is depicted in Fig. 2.b. In this case, as it has been shown in the literature, optical bistability also appears with some different properties than in previous case.

In both of these cases, the characteristics of the optical bistability obtained are strongly dependent on almost every one of the involved parameters. Each one may be a potential candidate to be taken as a quantity to be sensed if the whole structure is adopted as optical sensor. The basis of these different behaviors has been studied in several places being the main factor the different properties of the laser internal absorption when some parameter changes its value. Among the main applications of these configurations are the possibilities to arrange a large variety of architectures of interest in optical computing and modelling. This is due to the different Boolean functions able to be performed with them. This topic has been in the literature since more than twenty years ago.

Because the main objective of this paper is the potential application of these structures as basic elements for 


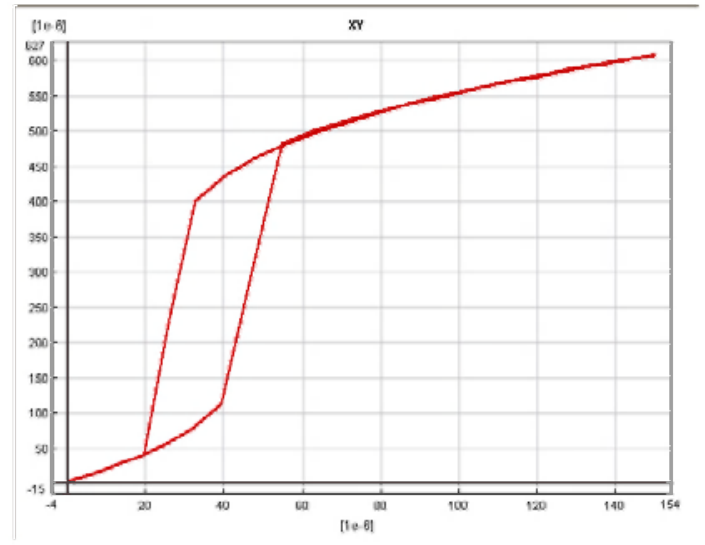

Fig. 3.- Optical bistable characteristics of the single laser diode structure working in the transmisive mode.

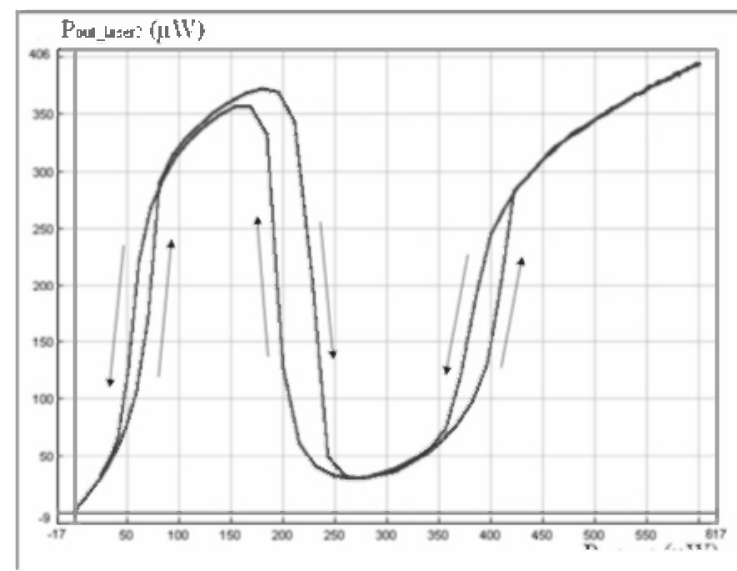

Figure 4.- Optical transfer function, $\left[\mathrm{P}_{\mathrm{n}}(\mathrm{uW}) . \mathrm{P}_{\text {out }}(\mu \mathrm{WW})\right]$ of the structure in Fig. 2 with a Fabry-Perot diode laser.

modeling sensing structures, we will concentrate here in the different responses obtained when some of their parameters modify their values and, as a consequence, the behavior changes too

The above reported structures have been studied previously by us and the main results may be seen in [6]-[8]. Because they are not the objective of this paper, we will ijust show here the main conclusions to be applied to our present objective. These results are summarized in Figs. 3 - 4. Fig. 3 shows the optical bistable characteristics of the composed structure of Fig. 1. It shows output light as a function of the input signal and the hysteresis cycle that offers. This twostates behaviour is characteristic of optical bistability. Moreover, its shape, position and main parameters values (switch-on and switch-off powers and hysteresis width) are strongly dependent on the wavelength of the impinging light and temperature. These will be the characteristics to be employed in our work.

Fig. 4 shows the working characteristics of the structures presented in Fig. 2. As it may be seen, two are now the

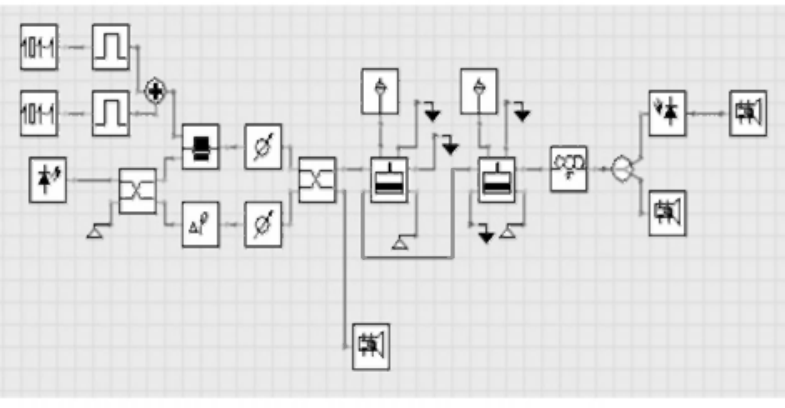

Figure 5.- Computer simulation of two semiconductor laser amplifiers with Fig. 4 as working behaviour.

states with high output light power as well as the number of regions with low light power. These results have been obtained, as well as the ones in Fig. 3, by computer simulation. The computer model appears in Fig. 5. As in the previous case, their properties are strongly dependent on several external parameters as temperature, wavelength on the incident light and detuning with respect to the proper frequency of the semiconductor laser amplifier.

\section{STRUCTURE OF THE FUNDAMENTAL NEURAL PROCESSING UNIT}

As it has been pointed out before, a simple cell has been the basis for most of the simulations of living beings sensory systems made by the authors. In everyone of the reported cases, this cell was partly based on the use of SelfElectrooptic Effect Devices, SEEDs, as non-linear element. These devices have the disadvantage of being scarcely used and no easily available in the market. Moreover, they do not have the possibility of an internal signal amplification. This fact reduces the fan-out of the system. It is because that we have employed Semiconductor Laser Amplifiers, SLA, in the present work. These components, having very strong non linearities as well as optical bistable properties, allows signal amplification and hence larger fan-out and the possibility to act onto a larger number of output devices.

The general configuration has been presented previously

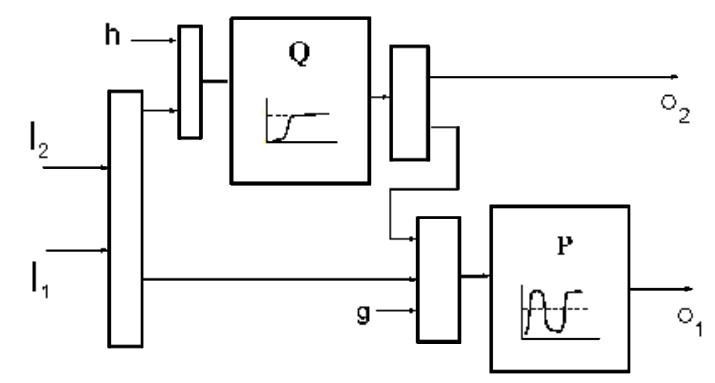

Figure 5.- Basic structure of the elemental block for the retina and visual cortex architectures. 
in several places [4]-[5]. The general structure appears in Fig. 5. Details may be seen in the literature. The main characteristic of this cell is the possibility process two input binary signals, governed by two control signals. Two outputs give logical functions of these inputs. The type of processing is related to the eight main Boolean Functions, namely, AND, OR, XOR, NAND, NOR, XNOR, ON and OFF. The programmable ability of the two outputs, as it has been described, allows performing a rather complete set of operations and, as a consequence, most of the functions performed by real neurons. Moreover, as it was shown, this circuit has the possibility to the generation of periodic and even chaotic solutions [6]-[7].

The main working conditions and results obtained from this cell are summarized in Table I.

TABLE I.

OUTPUT FUNCTIONS OF THE OPTICAL-PROGRAMMABLE LOGIC CIRCUIT

\begin{tabular}{|c|c|c|c|}
\hline $\begin{array}{c}\text { Q - Control } \\
\text { Signal } \rightarrow \\
\text { P - Control } \\
\text { Signal } \\
\downarrow\end{array}$ & $\begin{array}{l}\text { Q-output: } \\
\text { AND } \\
0-0.4 \\
\text { P - Output }\end{array}$ & $\begin{array}{c}\text { Q- Output: } \\
\text { OR } \\
0.5-0.9 \\
\text { P - Output }\end{array}$ & $\begin{array}{c}\text { Q-Output: } \\
\text { ON } \\
1.0-2.0 \\
\text { P - Output }\end{array}$ \\
\hline $0-0.4$ & $\mathrm{XOR}$ & XOR & NAND \\
\hline 0.5 & NAND & NOR & NOR \\
\hline $0.6-0.9$ & $\mathrm{ON}$ & XNOR & XNOR \\
\hline 1.0 & XNOR & XNOR & AND \\
\hline $1.1-1.4$ & XNOR & ON & OR \\
\hline 1.5 & AND & OR & OR \\
\hline $1.6-2.0$ & $\mathrm{OR}$ & OR & ON \\
\hline $2.0-2.5$ & ON & ON & ON \\
\hline
\end{tabular}

It is possible to see, from the results shown in Table I, that most of the main properties of single neurons, as well as those coming from groups of neurons, are possible to be obtained from the operations achieved by the present unit. As a matter of fact, the authors have reported previously the applications of this cell to the simulation of the five retinal layers and how the ganglion principal responses are obtained [4]-[5]. Moreover, these possible operations give a higher capability of simulation than the commonly used typical nonlinear activation operators as, for example, linear, unipolar hard limiter, unipolar sigmoidal, bipolar hard limiter, piecewise linear, bipolar sigmoidal or multimodal operators. The reported cell is able to perform any one of these functions just by choosing the adequate control parameters. This possibility opens a new form of modelling neural networks with just a single type of cell. At the same time, because the optical bistable properties of this structure, a periodic behaviour may be also obtained. This makes possible to handle similar responses as those had by neurons as amacrine or ganglion cells in the mammalian visual system.

Some of these properties were reported previously [10] but one of the nonlinear devices employed was a SEED. As we have indicated, some advantages may be obtained by using SLAs instead. The more important one is that the number of layers following to a cell is not limited by power. This is due to the amplification given by SLAs. Hence the number of connections to other neurons is not limited and a single unit may act on a larger number of cells.

\section{CONCLUSIONS}

A new type of fundamental cell, able to simulate any behavior of biological neurons, has been reported. It is based on the use of semiconductor laser amplifiers as nonlinear elements with optical bistable properties. Its main advantage is its large fan-out, larger than with any other nonlinear device, and the possibility to be implemented with very common devices. Moreover, these units may be employed with structures employed in other fields, as Optical Communications were the number and properties of existing components is very large. This gives a substantial improvement with respect to previously employed structures. Some applications have been developed and some others are under study and will be presented.

\section{ACKNOWLEDGMENT}

This work was partly supported by CICYT, grant TIC200304309

\section{REFERENCES}

1. Hopfield, J.J., "Neural networks and physical systems with emergent collective computational abilities", Proceedings of the National Academy of Sciences 79, 2554-2558 (1982).

2. See, for example, F.T.S. Yu and S. Jatamulia, "Optical Signal Processing, Computing, and Neural Networks", Wiley (1992).

3. S. Suzuki et al., "An Experiment on High-Speed Optical Time-Division Switching". Journal of Lightwave Technology, vol. 4, pp. $894-99$ (1986)

4. J.A. Martín-Pereda, A. González-Marcos and C. Sánchez-Guillén, "A model of the Mammalian Retina and the Visual Cortex. Disorders of vision". In Proc. IEEE Conference on Engineering in Medicine and Biology. Technical Digest CD-ROM 138-140. Amsterdam, 1996.

5. A. González-Marcos \& J.A. Martín-Pereda, "A New Approach to a Model of the Mammalian Retina with Optically Programmable Logic Cells". $1^{\text {st }}$. International IEEE EMBS Conference on Neural Engineering. Capri (Italia). 20-22 de marzo, 2003.

6. A. González-Marcos and J.A. Martín-Pereda, "Digital chaotic output from an optically processing element", Optical Engineering, vol. 35, pp. 525-535, 1996

7. A. González-Marcos and J.A. Martín-Pereda, "Analysis of irregular behaviour on an optical computing logic cell". Optics \& Laser Technology, vol. 32, pp. $457-466,2000$.

8. A. Hurtado, A. Gonzalez-Marcos and J.A. Martin-Pereda, "Modeling Reflective Bistability in Vertical-Cavity Semiconductor Optical Amplifiers", IEEE Journal of Quantum Electronics. (Accepted for publication)

9. A. González-Marcos and J.A. Martín-Pereda, "Method to analyze the influence of hysteresis in optical arithmetic units". Optical Engineering, vol. 40, pp. 2371-2385, 2001

10. J.A. Martín-Pereda and A. González-Marcos, "Image processing based on a model of the mammalian retina". In Applications of Photonics Technology 3. Edited by G.A.Lampropoulos and R.A. Lessard. SPIE Volume 3491, 1185-1190, 1998 